\title{
Abdominal trauma: a report of 129 cases
}

\author{
T. BATES* \\ F.R.C.S. \\ Luton and Dunstable Hospital, Luton, Bedfordshire
}

\begin{abstract}
Summary
A retrospective study of 129 cases of abdominal trauma admitted to a district general hospital over the 8 years 1964-71 is reported. Road traffic accidents accounted for $60 \%$ of the cases and had a much higher mortality than domestic or industrial accidents.

Laparotomy was carried out in eighty-eight patients, but two patients with a ruptured abdominal viscus died without operation because the diagnosis was not recognized.

There were seventy-four cases of renal injury of which thirty-nine were treated conservatively and thirty-four were explored through a laparotomy incision. The indication for urgent operation in every case was the suspicion of an associated intraperitoneal injury and in all but three this was confirmed. Only one injured kidney was explored through the loin after an interval. Nephrectomy was carried out in eight cases (11\%).
\end{abstract}

The commonest finding at laparotomy was rupture of the spleen, of which there were fifty-three cases. Major hepatic injuries and rupture of the duodenum carried a very high mortality. In all four cases of retroperitoneal rupture of the duodenum there was a delay in diagnosis of at least $24 \mathrm{hr}$ due to the late onset of physical signs.

The overall mortality of patients with proved rupture of an abdominal viscus was $17 \%$ but in twenty patients $(22 \%)$ there was a delay in diagnosis and this group carried a $30 \%$ mortality.

A diagnostic peritoneal tap was carried out in only fifteen cases, but in nine $(60 \%)$ gave a false negative result.

The place of diagnostic peritoneal lavage in the management of abdominal trauma is discussed.

\section{Introduction}

Injury of the abdomen accounts for a very small proportion of the injuries in accident cases (Gögler, 1965) and patients who have ruptured an abdominal viscus often have other injuries which may mask the abdominal signs and symptoms. Improved techniques of resuscitation and intensive postoperative care have improved the prognosis of such injuries (Welch \& Giddings, 1950) but delay or failure in

\footnotetext{
* Present address: St Olave's Hospital, Lower Road, Rotherhithe, London SE16 2TS.
}

diagnosis still remains an important factor in contributing to the high mortality of abdominal trauma (Tovee, 1970).

The Luton and Dunstable Hospital is a district general hospital which receives a relatively large number of severe abdominal injuries because of its proximity to the busiest part of the M1 motorway.

This paper records the pattern of injury following abdominal trauma in patients admitted to this hospital in the 8 years 1964-71. The causes and mortality of abdominal trauma are also discussed together with the use of various diagnostic techniques. Patients who suffered a ruptured abdominal viscus or renal injuries are considered, but those who were successfully treated conservatively for other abdominal trauma are not included in this study.

\section{Results}

During the 8 years under review, 129 patients were admitted to the Luton and Dunstable Hospital who required a laparotomy or management of renal injury following abdominal trauma. Laparotomy was carried out in eighty-eight patients, and a further two patients died without laparotomy because rupture of an abdominal viscus was not diagnosed.

There were seventy-four patients with evidence of renal injury of which thirty-four had an urgent laparotomy carried out and thirty-nine were treated conservatively. In only one case was an injured kidney explored through the loin, and this was after an interval.

The most common finding at laparotomy (Table 1) was rupture of the spleen (fifty-three cases), but this was the sole injury in only twenty-one cases. There were five deaths in the seventeen patients with a ruptured liver, but four occurred in the seven patients with major hepatic injury.

Four out of five patients with rupture of the duodenum died, and in three this was largely due to delay in diagnosis. Rupture of the small bowel occurred in six cases, and there were several injuries of the mesentery, colon and bladder. There was no case of injury to the biliary tract or severe pancreatic injury.

Associated extra-abdominal injuries (Table 2) occurred in fifty-eight $(45 \%)$ of the cases, of which chest injuries $(30 \%)$ were the most common. Fifteen deaths occurred in this series of which eleven (73\%) had one or more associated extra-abdominal injuries. 
TABle 1. Abdominal injuries in 129 cases

\begin{tabular}{lrccc}
\hline \multicolumn{1}{c}{ Site } & No. & $\begin{array}{c}\text { Incidence } \\
\%\end{array}$ & Deaths & $\begin{array}{c}\text { Mortality } \\
\%\end{array}$ \\
\hline Kidney & 74 & 57 & 4 & 5 \\
Spleen & 53 & 41 & 8 & 15 \\
Liver & 17 & 13 & 5 & 29 \\
Small bowel & 8 & - & 1 & - \\
Mesentery & 7 & - & 1 & - \\
Duodenum & 5 & - & 4 & 80 \\
Pancreas & 4 & - & - & - \\
Bladder & 3 & - & - & - \\
Colon & 2 & - & 1 & - \\
Abdominal wall only & 2 & - & - & - \\
Stomach & 1 & - & - & - \\
Diaphragms & 1 & - & 1 & - \\
Ureter & 1 & - & - & - \\
\hline \multicolumn{1}{c}{ Total } & 129 & - & 15 & 12 \\
\hline
\end{tabular}

TABlE 2. Associated injuries in 129 patients with abdominal injuries

\begin{tabular}{|c|c|}
\hline Injury & No. \\
\hline Fractured ribs & $27\}(30 \%)$ \\
\hline Severe limb injuries & $20(15 \%)$ \\
\hline Head injury & $14(11 \%)$ \\
\hline Fractured pelvis & 6 \\
\hline Ruptured urethra & 2 \\
\hline Total & $58 \quad(45 \%)$ \\
\hline
\end{tabular}

In six of the fatal cases there was a delay in the diagnosis of a ruptured abdominal viscus of $24 \mathrm{hr}$ or more and in four of these the delay was largely attributable to associated injuries.

In three cases there was delayed rupture of a subcapsular splenic haematoma, but in twenty cases $(22 \%)$ there was a delay in diagnosis of $6 \mathrm{hr}$ or more and this group carried a $30 \%$ mortality. The overall mortality in this series of 129 cases was $12 \%$.

There was no death in the thirty-nine patients treated conservatively for renal injuries, and if these are excluded the mortality of a ruptured abdominal viscus was $17 \%$.

\section{Causes of abdominal trauma (Table 3)}

Seventy-eight $(60 \%)$ of the abdominal injuries resulted from road traffic accidents and the remaining fifty-one cases $(40 \%)$ followed accidents at home, work or sport, or were caused by fights. Of those injured in road accidents, $64 \%$ were vehicle occupants. However, of twenty-four children under the age of 15 years, fourteen $(58 \%)$ were pedestrians or pedal cyclists.

There were only four penetrating abdominal injuries, three of which were from stab wounds. All were explored but in two no intraperitoneal injury was found on laparotomy.
TABLE 3. Causes of abdominal injury (129 cases)

\begin{tabular}{lr}
\hline Road traffic accidents $(60 \%)$ & \\
Vehicle occupants & 50 \\
Pedestrians & 11 \\
Motor cycle/scooter riders & 9 \\
Pedal cyclists & $\underline{8}$ \\
& $\underline{78}$ \\
Other injuries (40\%) & 18 (6 at work) \\
Falls from height & 13 (10 at work) \\
Crush injuries & 10 \\
Sport & 4 \\
Fights (blunt injury) & 3 \\
Stabs & 3 \\
Equestrian & 51 \\
\hline
\end{tabular}

Of the fifteen deaths in the series, all except one followed road accidents. Associated injuries occurred in fifty-two $(67 \%)$ of those injured in road accidents but in only twelve cases $(24 \%)$ injured in other accidents. It is evident from both the mortality and the high incidence of associated injuries that the severity of blunt abdominal trauma in road accidents is, on the whole, greater than in other accidents. It is noted that abdominal and other injuries from motorway accidents carried a higher mortality (Table 4) than those from other road accidents.

TABLE 4. Casualties from road traffic accidents

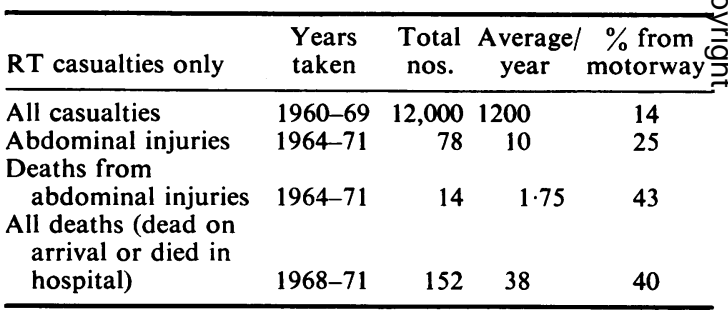

\section{Ruptured spleen (fifty-three cases)}

There were fifty-three cases of ruptured spleen. Thirty-four $(64 \%)$ were males between the age of 3 and 30.

McIndoe (1932) arbitrarily defined delayed rupture of the spleen as one occurring more than $48 \mathrm{hr}$ after injury. Using this definition there were seven such cases $(13 \%)$, comparable with the incidence of $14 \%$ found by Zabinski \& Harkins (1943) in a review of 171 cases.

However, in a further ten patients operation was delayed for between 6 and $48 \mathrm{hr}$. Two patients with splenic rupture and associated injuries died after $24 \mathrm{hr}$ without laparotomy because the diagnosis was missed.

Thus, of fifty-three patients, only thirty-four $(64 \%)$ had physical signs diagnostic of a ruptured spleen on admission. Only three of those with delayed rupture had evidence of subcapsular haematoma which had 
subsequently ruptured. Several had a mass of clots surrounding the spleen suggesting that local tamponade had slowed the rate of bleeding, but in the majority there was no evidence of such a mechanism.

Thirty-six of the fifty-three patients with a ruptured spleen had X-rays available for review, and of these twenty-one $(58 \%)$ had appearances of diagnostic value. Eight had a fracture of a left lower rib and the same number had an opacity in the left upper quadrant. The left diaphragm was raised in five cases, but convincing loss of the left psoas shadow or displacement of the gastric gas shadow was only seen in three cases.

There was some degree of haematuria in twenty-six $(49 \%)$ cases with splenic rupture. There was proved renal contusion or rupture in seventeen of the fiftythree cases $(32 \%)$.

Stivelman, Glaubitz \& Crampton (1963) noted evidence of renal contusion in only $7 \%$ of 100 cases of ruptured spleen. However, Parsons \& Thompson (1958) found renal contusion or rupture in $43 \%$ of their cases.

The post-operative complications of splenectomy are well recognized (Parsons \& Thompson, 1958), pulmonary complications being the most common. Clarke (1954) found chest complications in $41 \%$ of cases following splenectomy for trauma. In the present series sixteen $(30 \%)$ had a chest infection or basal collapse. Three patients had clinical evidence of a pulmonary embolus although only one patient was noted to have a deep venous thrombosis (DVT). One suspects that the true incidence of DVT was far higher and that some of the pulmonary complications were due to infarction rather than infection (Irving et al., 1973).

Two patients developed a subphrenic abscess and four had a wound dehiscence or late incisional hernia. There was no case of pancreatic fistula.

Three further patients had a minor splenic capsular tear. Two were noted at operation and left alone, and a third posterior capsular tear was missed at laparotomy. All three patients subsequently died of other injuries but at necropsy none had bled significantly from the spleen.

There were eight deaths in the fifty-three cases of ruptured spleen ( $15 \%$ mortality), whereas in the twenty-one cases in which this was the sole significant injury there were only two deaths $(9.5 \%$ mortality). The mortality of the thirty-two cases with associated injuries was $19 \%$.

In a review of the literature, Woolridge (1969) found the reported mortality following splenectomy for trauma varied between 0 and $33 \%$. The average mortality was $15 \%$ and the greater the degree of other injuries, the greater the mortality.

Stivelman, Glaubitz \& Crampton (1963) found a lower mortality in delayed rupture of the spleen
$(6.6 \%)$, but Foster (1970) in a review of the literature found an increase in this group. Two of the seven patients with delayed rupture died, giving an increased mortality of $29 \%$ in this series.

\section{Injuries to the liver (seventeen cases)}

Blumgart \& Vajrabukka (1972) have defined minor liver injury as one that can be closed to its full depth by simple suture with control of haemorrhage and little associated parenchymal damage. Using this classification there were ten minor and seven major injuries of the liver.

All the major liver injuries involved the right lobe, although there were four minor lacerations involving the left lobe. There was seldom much difficulty in approximating the edges of minor lacerations and the bleeding had often stopped spontaneously. Minor liver injuries were never the sole significant injury and the only death in this group was from multiple associated injuries.

In two patients with major liver injuries a right hemihepatectomy was carried out for uncontrollable bleeding. In one case this procedure failed to control the bleeding; the other patient died with portal vein thrombosis after 8 days.

A further patient who had extensive debridement to a rupture of the right lobe associated with a rupture of the right diaphragm subsequently died of a secondary haemorrhage from an unrecognized tear in the portal vein. There were only three survivors from major liver injuries and all were treated with conservative debridement.

In no case was a pack left in the liver at the end of the operation, nor was the biliary tract drained.

There were associated injuries in sixteen of the seventeen cases $(94 \%)$. Ten patients had splenic rupture and three required nephrectomy. The overall mortality of ruptured liver in this series was $29 \%$, but when major liver injuries alone are considered the mortality rose to $57 \%$. Little \& Williams (1969) found an overall mortality of $24 \%$, but Clarke (1954) had a $50 \%$ mortality in eighteen cases.

\section{Rupture of the duodenum (five cases)}

There were five cases of rupture of the duodenum. In one case there was a complete transection at the duodeno-jejunal flexure which was treated by end-toend anastomosis. This was the only injury to the duodenum recognized as a ruptured viscus on admission, but the patient died of haemorrhage from an acute gastric erosion 8 days post-operatively. The anastomosis was intact at post-mortem examination.

The other four cases of duodenal rupture involved the retroperitoneal part and all were at or near the junction of the second and third parts of the duodenum. There was a delay in diagnosis of between 
24 and $48 \mathrm{hr}$ in all these patients, and only one survived.

Two patients developed a duodenal fistula following surgical repair carried out $24 \mathrm{hr}$ after injury. In one case this persisted in spite of two further attempts at closure and the patient died 3 months later with recurrent local sepsis. The other duodenal fistula closed spontaneously and the patient survived.

The other two patients with retroperitoneal rupture of the duodenum who died had free intraperitoneal fluid as well as marked retroperitoneal bile-staining of the mesentery and mesocolon.

\section{Mesenteric and small bowel injuries (fifteen cases)}

There were eight cases of small bowel rupture, five of which had a perforation of the proximal jejunum between 7 and 10 in. below the duodeno-jejunal flexure. Most of these ruptures were on the antimesenteric border and presumably occurred at a point where the jejunum was compressed against the lumbar spine. One patient had a gangrenous segment of ileum due to a transverse mesenteric laceration which required resection. There were six other cases with one or more lacerations in the mesentery, mesocolon or mesosigmoid, but none of these caused significant bowel ischaemia.

One patient developed intestinal obstruction 1 week after an abdominal blow and was found to have a tumour-like mass obstructing the mid-ileum. This was resected and histology showed a non-specific stenosis due to haemorrhage and fibrosis. A similar obstruction to the jejunum is described by Lampert, Goodfellow \& Wachowski (1954) and duodenal obstruction due to intra-mural haematoma is well recognized (Mathewson \& Morgan, 1966). There was a delay in diagnosis in three patients with rupture of the small bowel of between 8 and $24 \mathrm{hr}$, and one subsequently died.

\section{Colonic injuries (two cases)}

Two patients sustained a rupture of the colon, although several others were noted to have a traumatic myotomy. One patient had a small perforation of the splenic flexure associated with a rupture of the spleen. This was treated by simple suture and the patient survived.

A second patient suffered a penetrating abdominal wound with transection of the sigmoid colon in a road traffic accident which was treated by a terminal colostomy with closure of the rectal stump. He died of a massive pulmonary embolus $\mathbf{1 0}$ days later.

\section{Renal injuries (seventy-four cases)}

There were thirty-eight patients with gross haematuria following abdominal trauma, all of whom had an emergency intravenous pyelogram (IVP). Twentyseven were treated conservatively and ten had an urgent laparotomy for signs of haemoperitoneum and shock. In only one case in this series was an injured kidney explored through the loin and this was done after an interval. The injured kidney did not function on IVP in three of the patients who were explored and all came to nephrectomy. In two cases there was gross rupture of the kidney and in the third there was traumatic rupture of a Wilm's tumour in a boy aged 4 .

In the other seven patients with gross haematuria who were urgently explored there was rupture of the spleen with contusion of the left kidney. In five of these cases an IVP was normal, but the other two showed minor injury to a solitary functioning left kidney. A further patient who injured a longstanding hydronephrosis came to nephrectomy after 3 days when an attempted pyeloplasty for increasing obstruction failed.

Nine of the twenty-seven patients with gross haematuria who were treated conservatively had a normal IVP and nine had intrapelvic or calyceal clots which subsequently resolved. In the other nine patients there was grossly reduced function or a calyceal leak from the injured kidney. Five of these returned to normal function with minimal scarring but in two cases there was loss of function in one pole of the kidney. Two were lost to follow-up.

Four further patients had a nephrectomy carried out at an emergency laparotomy for abdominal injuries. In one case the left kidney was avulsed and lying free within the left chest and in the other three the kidney was ruptured into two or three parts and was judged to be irreparable. No record of haematuria was made in these four patients and none had an IVP although this investigation may have been prevented by the state of shock on admission in at least two of the cases. Nineteen $(45 \%)$ of the fortytwo patients with gross haematuria or a ruptured kidney had one or more associated injuries (Table 5).

There were thirty-two patients with microscopic haematuria and in twelve this was the sole evidence of renal injury and was treated conservatively. Microscopic haematuria was also noted in twenty patients who had a laparotomy for abdominal

TABLE 5. Associated injuries in forty-two patients with gross haematuria or ruptured kidney

\begin{tabular}{lrl}
\hline \multicolumn{1}{c}{ Injury } & No. \\
\hline Fractured lower ribs & & \\
on side of injury & 13 & $(31 \%)$ \\
Ruptured spleen & 9 & $(21 \%)$ \\
Head & 5 & \\
Chest & 5 & \\
Ruptured liver & 5 & \\
Limb & 3 & \\
Ruptured diaphragm & 1 & \\
\hline Total & 19 & $(45 \%)$ \\
\hline
\end{tabular}


injuries. It is noteworthy that in seventeen cases the associated injury was a rupture of the spleen and that in most of these there was contusion of the left kidney or a perirenal haematoma.

Four patients with renal injuries died, but in each case this was attributable to severe associated injuries. Thus the mortality of renal injury itself was very low.

It was not found possible to carry out repair or partial nephrectomy in any ruptured kidney, and although an approach through the loin might have made conservative surgery practicable this was contraindicated by the high incidence of associated intraperitoneal injuries. Nephrectomy was carried out in eight cases ( $11 \%$ ), but if the ruptured Wilm's tumour is excluded, the nephrectomy rate in this series was $9.5 \%$.

There was one case of partial rupture of the upper third of the left ureter which healed without stricture following simple extraperitoneal drainage. This was caused by a direct blow and was associated with fractures of the transverse processes of L3 and L4.

There were two cases of intraperitoneal rupture of the bladder. In one the diagnosis was delayed for 2 weeks because the small tear at the vault caused only a partial leak and failed to show on an initial IVP or subsequent micturating cystogram.

There was one case of extraperitoneal rupture of the bladder in which there was no pelvic fracture, and it is also unusual that both the intraperitoneal ruptures were associated with a fractured pelvis.

Rupture of the membranous urethra was found in two patients with rupture of the spleen and pelvic fractures, but urethral injuries have not been considered in this study.

\section{Associated injuries}

There were associated extra-abdominal injuries in fifty-eight $(45 \%)$ of the cases. $30 \%$ had chest injuries and $20 \%$ had severe skull or limb fractures.

Abdominal injuries may be overlooked when there are severe head, chest or limb injuries, and injuries of the pelvis, costal margin or lumbar spine present particular difficulties in diagnosis. Two patients in this series had a general anaesthetic for limb fractures soon after admission and their abdominal injuries did not become apparent for $24 \mathrm{hr}$. Both patients died.

On the other hand, associated injuries may be missed when the patient obviously needs an urgent laparotomy for abdominal injuries. One patient with a ruptured liver and spleen became shocked and continued to deteriorate soon after admission in spite of rapid transfusion. She was noted to have several fractured ribs and her condition improved dramatically when she was anaesthetized. It was not until she resumed spontaneous respiration at the end of the operation that a flail chest with paradoxical movement was noticed. She required positive pressure ventilation for 2 weeks and recovered.

\section{Diagnostic peritoneal taps and peritoneal lavage}

Four-quadrant peritoneal taps were carried out on fifteen patients. Subsequently proved haemoperitoneum was shown in five cases, and there was a negative tap following a renal injury which was successfully treated conservatively.

However, in nine patients $(60 \%)$ there was a false negative tap. In seven cases there was a ruptured spleen and at laparotomy shortly afterwards five of these had a measured haemoperitoneum of $1 \frac{1}{2}-31$. In no case did a false negative tap delay operation, but in three unconscious patients diagnostic peritoneal lavage, carried out after a negative tap, demonstrated a haemoperitoneum which in each case was due to a ruptured spleen.

In order to carry out diagnostic lavage, a subumbilical dialysis catheter is introduced into the peritoneum, providing there is no abdominal scar, and 1 litre of normal saline is instilled. The patient is tipped head down before the irrigating fluid is run out. A crystal-clear return virtually excludes a significant haemoperitoneum or intraperitoneal rupture of the bowel, but cannot exclude a true subcapsular haematoma of the spleen or retroperitoneal rupture of the duodenum. Microscopy of the irrigating fluid may be of some value. This technique was only used in three cases in this series but has also been used in twenty further cases of abdominal trauma without giving a false result.

\section{Discussion}

The overall mortality in this series was $12 \%$ and although comparison with other series of abdominal trauma is difficult since different groups are considered, this is similar to the $13 \%$ found both by Morton, Roys \& Bricker (1972) and by Welch \& Giddings (1950). The latter authors, however, found that the mortality in their series of 200 patients dropped from $19 \%$ in the early years to $3 \%$ in the latter part.

Morton, Hinshaw \& Morton (1957) found nine fatal cases of missed abdominal injury in a series of 120. There were two such patients in this series and there are few large reviews which do not show a similar experience.

Delay in diagnosis of ruptured spleen, and more especially rupture of the duodenum, carried a high mortality in this series. In many cases in which rupture of the spleen is said to be delayed, the delay is probably one of diagnosis rather than of rupture. It is felt that the ability of fit young men to compensate adequately for severe intraperitoneal haemorrhage, and for many hours, has been insufficiently stressed in the past. 
The four cases of retroperitoneal rupture of the duodenum in this series re-emphasize the sinister reputation of this injury in which the onset of gross physical signs may be delayed for $24 \mathrm{hr}$ or more. The classical appearance of retroperitoneal gas in the right hypochondrium is often absent or its presence missed. It was noted in only one of the present cases. The use of contrast medium in the radiological diagnosis of this injury may have a limited place, but if not available urgent laparotomy should be carried out on the least suspicion.

Delay in the diagnosis of retroperitoneal rupture of the duodenum is well recognized (Cohn, Hawthorne \& Frobese, 1952; Rothchild \& Hinshaw, 1956), and the lesion may easily be missed at laparotomy (Newell, Rosenbaum \& Canter, 1951).

Wilson \& Costopoulos (1971) reviewing six cases of retroperitoneal rupture of the duodenum found that all were initially misdiagnosed, the average delay in diagnosis being $26 \mathrm{hr}$ (12-60). Three of their patients had free bile-stained fluid in the peritoneal cavity and they postulated that this leakage probably occurred at the time of the patients' deterioration, since the retroperitoneal tissues were also bile stained.

Intravenous or jejunostomy feeding has an important part to play in the management of those patients who subsequently develop a duodenal fistula, and there is much to be said for placing a jejunostomy tube at the time of repair.

There were only seven major liver injuries, which carried a very high mortality. Massive hepatic resection for major liver injury is widely practised (McClelland \& Shires, 1965). However, Morton, Roys \& Bricker (1972) have recently shown, in a series of over 1000 cases, that a more conservative approach does not raise the mortality of hepatic rupture. Biliary decompression has been advocated by Merendino, Dillard \& Cammock (1963) and is commonly used following major hepatic resection. However, Lucas (1971) has recently thrown some doubt on its advisability.

Renal angiography was not used in the investigation of renal injury in this series, although Elkin, Chien-Hsing Meng \& de Paredes (1966) and Solheim, Resch \& Kordt (1972) have strongly advocated its use in the emergency situation. On the other hand, Slade (1971) argued that a more conservative approach is satisfactory, using an emergency IVP and the clinical criteria of continued, profuse haematuria, or an increasing loin swelling with shock, as an indication for exploration. However, the indication for urgent exploration in every case, in this series, was the suspicion of associated intraperitoneal injury. For this reason a laparotomy was always carried out and a ruptured abdominal viscus was confirmed in thirty-one of the thirty-four cases explored, and in four of the seven cases in which emergency nephrectomy was carried out. Both these latter series had an eventual nephrectomy rate of $8 \%$, the comparable figure in the present series being $9.5 \%$.

Slade (1971) considers antibiotics mandatory following renal injury but although they were used in less than a third of patients in the present series there was no case of infection. Neither was any case of late hypertension found, although only twenty patients with more severe renal injuries were followed up from 1 to 3 years.

Removal of a solitary kidney is a rare but avoidable disaster (Welch \& Giddings, 1950). Two patients with gross haematuria were found to have an injury to a solitary functioning kidney on pre-operative emergency IVP. In both cases this amounted to no more than a contusion of the kidney, but in four other cases nephrectomy was carried out without pyelographic evidence of function in the contralateral kidney. It is felt that if a patient requires a laparotomy for abdominal trauma and is unable to pass urine, and there is no evidence of urethral injury, a urethral catheter should be passed. If this demonstrates haematuria a limited IVP is essential and takes at most $10 \mathrm{~min}$ if carried out at the same time as other essential X-rays. Furthermore, in the seriously injured patient who has had a period of hypotension before adequate transfusion, knowledge of the urinary output in the post-operative period is essential. Two patients in this series died of renal failure and a third required temporary dialysis following a hypotensive episode.

Pontius, Kilbourne \& Paul (1956) found associated injuries in $30 \%$ of their cases of abdominal injury and point out the diagnostic pitfalls and increased mortality of this group. In the present series associated injuries sometimes caused delay in diagnosis of a ruptured abdominal viscus, and both these factors increased the mortality of abdominal injury. A thorough examination should be carried out at the time of initial assessment and the priority of any given injury carefully considered. It is felt that a routine X-ray of the chest and pelvis should be carried out in nearly all cases of abdominal trauma in addition to any other $\mathrm{X}$-rays indicated by injuries found on examination. The presence of bruising or marking from clothes on the anterior abdominal wall usually indicates significant abdominal trauma.

The use of four-quadrant peritoneal taps in the diagnosis of abdominal injuries is still controversial, and diagnostic peritoneal lavage is not widely used in this country. Byrne (1956) claimed an $83 \%$ accuracy for peritoneal taps, but Brittain (1963) found false negative taps in $65 \%$ of fifty-one cases of abdominal injury. The small number of taps in the present series does not include several patients who 
had a negative tap and were successfully treated conservatively, but the incidence of false negative taps does point to the serious limitations of the method.

Perry, de Meules \& Root (1970) found diagnostic peritoneal lavage accurate in $96 \%$ of 304 cases. This method has been used in only twenty-three cases of abdominal trauma but has given the correct result in each case and has not caused any complication.

Occasional perforation of the bowel has been reported following the use of an introducer for the insertion of a peritoneal dialysis catheter, and for this reason Perry, de Meules \& Root (1970) now advocate formal placement of the catheter into the peritoneum. An adjacent abdominal scar contraindicates the use of a trocar or introducer.

Simple abdominal paracentesis has recently been advocated (Gjessing et al., 1972), but having placed a dialysis catheter in the peritoneum (which is more easily done with an introducer than a trocar and cannula), it is a simple matter to carry out a thorough diagnostic lavage and the chance of obtaining a false negative would seem much less.

Careful and repeated clinical examination must remain the chief criterion for laparotomy in cases of suspected abdominal, injury. However, whilst there is no point in carrying out either a peritoneal tap or diagnostic lavage in a patient who obviously needs an urgent laparotomy, the high incidence of delay or failure in diagnosis in this and many other series of abdominal trauma points to the need for such investigation in those cases in which there is some clinical doubt but not enough to warrant laparotomy. Perhaps diagnostic peritoneal lavage should supercede simple tap in such cases.

A negative laparotomy for abdominal trauma was only carried out on two occasions in the 8 years under review. This compares unfavourably with the number of cases in which operation was delayed or omitted. The reluctance to submit a patient with severe associated injuries to a laparotomy on minimal clinical grounds undoubtedly contributes to the high mortality of abdominal trauma.

\section{Acknowledgments}

I would like to thank the constulant surgeons of the Luton and Dunstable Hospital (Mr D. H. C. Harland, Mr R. V. Fiddian, Mr W. M. Mee and Mr R. L. Rothwell-Jackson) for allowing me to review patients under their care and $\mathrm{Mr}$ M. H. Irving and Mr R. V. Fiddian for help in preparing this paper. I would also like to thank Miss D. E. Nicholls and Miss J. M. Stephen for collecting the records. I am very grateful to $\mathrm{Mr}$ J. F. Hindle and H.M. Coroner for South Bedfordshire for providing the information in Table 4 .

\section{References}

Blumgart, L.H. \& VAJRabukKa, T. (1972) Injuries to the liver: analysis of twenty cases. British Medical Journal, 1, 158.
BRITTAIN, R.S. (1963) Liver trauma. Surgical Clinics of North America, 43, 433.

ByrNe, R.V. (1956) Diagnostic abdominal tap. Western Journal of Surgery, 64, 369.

Clarke, R. (1954) Closed abdominal injuries. Lancet, ii, 877.

Cohn, I., Hawthorne, H.R. \& Frobese, A.S. (1952) Retroperitoneal rupture of duodenum in non-penetrating abdominal trauma. American Journal of Surgery, 84, 293.

Elkin, M., Chien-Hsing Meng \& De Paredes, R.G. (1966) Roentgenologic evaluation of renal trauma with emphasis on renal angiography. American Journal of Roentgenology, 98, 1.

Foster, R. P. (1970) Delayed haemorrhage from the ruptured spleen. British Journal of Surgery, 57, 189.

Gjessing, J., Oskarsson, B.M., Tomlin, P.J. \& Brock-Utne, J. (1972) Diagnostic abdominal paracentesis. British Medical Journal, 1, 617.

Gögler, E. (1965) In: Series chirurgica, Road Accidents, p. 33. Documenta Geigy, Basle.

Irving, M.H., Payne, R.A., O’Brien, E.N. \& Wilks, R. (1973). In press.

LAMPeRT, E.G., Goodfellow, J.G. \& WAChOWSKI, T.J. (1954) Traumatic subserosal haemorrhage causing small bowel obstruction. Annals of Surgery, 140, 768.

LitTle, J.M. \& Williams, C.W. (1969) Improved mortality in the management of liver injuries. British Journal of Surgery, 56, 603.

LuCAs, C.E. (1971) Prospective clinical evaluation of biliary drainage in hepatic trauma: an interim report. Annals of Surgery, 174, 830 .

McClelland, R.N. \& Shires, T. (1965) Management of liver trauma in 259 consecutive patients. Annals of Surgery, 161, 248.

MCINDOE, A.H. (1932) Delayed haemorrhage following traumatic rupture of the spleen. British Journal of Surgery, 20, 249.

Mathewson, C. \& Morgan, R. (1966) Intramural haematoma of the duodenum: a clinical entity. American Journal of Surgery, 112, 299.

Merendino, K.A., Dillard, D.H. \& Cammock, E.E. (1963) The concept of surgical biliary decompression in the management of liver trauma. Surgery, Gynaecology and Obstetrics, 117, 285.

Morton, J.H., Hinshaw, J.R. \& Morton, J.J. (1957) Blunt trauma to the abdomen. Annals of Surgery, 145, 699.

Morton, J.R., Roys, G.D. \& Bricker, D.L. (1972) The treatment of liver injuries. Surgery, Gynaecology and Obstetrics, 134, 298.

Newell, R.B., Rosenbaum, G.R. \& Canter, N.M. (1951) Traumatic retroperitoneal rupture of the duodenum. Annals of Surgery, 134, 277.

Parsons, L. \& Thompson, J.E. (1958) Rupture of the spleen from nonpenetrating injuries. Annals of Surgery, 147, 214.

Perry, J.F., De Meules, J.E. \& Root, H.D. (1970) Diagnostic peritoneal lavage in blunt abdominal trauma. Surgery, Gynaecology and Obstetrics, 131, 742.

Pontius, G.V., Kilbourne, B.C. \& Paul, E.G. (1956) Nonpenetrating abdominal trauma. Archives of Surgery, 72, 800.

RothChild, T.P.E. \& Hinshaw, A.H. (1956) Retroperitoneal rupture of the duodenum caused by blunt trauma with a case report. Annals of Surgery, 143, 269.

SLADE, N. (1971) Management of closed renal injuries. British Journal of Urology, 43, 639.

Solmeim, K., Resch, F. \& KordT, K.F. (1972) Closed renal injuries. Injury, 3, 185.

Stivelman, R.L., Glaubitz, J.P. \& Crampton, R.S. (1963) Laceration of the spleen due to nonpenetrating trauma. American Journal of Surgery, 106, 888.

ToveE, E.B. (1970) Blunt abdominal trauma. Journal of Trauma, 10, 72 . 
Welch, C.E. \& Giddings, W.P. (1950) Abdominal trauma: a clinical study of 200 consecutive cases from the Massachusetts General Hospital. American Journal of Surgery, 79, 252.

Wilson, T.S. \& Costopoulos, L.B. (1971) Retroperitoneal injury to the duodenum by blunt abdominal trauma: a report of eight cases. Canadian Journal of Surgery, 14, 114 WoolRIDGE, B.F. (1969) Traumatic rupture of the spleen. Missouri Medicine, 66, 804.

ZABINSKI, E.J. \& HARKINS, H.N. (1943) Delayed splenic rupture: a clinical syndrome following trauma. Archives of Surgery, 46, 186. 